\title{
Fuzzball solutions for black holes and D1-brane-D5-brane microstates
}

\author{
Kostas Skenderis* and Marika Taylor* \\ Institute for Theoretical Physics, University of Amsterdam, \\ Valckenierstraat 65, 1018 XE Amsterdam, The Netherlands.
}

\begin{abstract}
We revisit the relation between fuzzball solutions and D1-D5 microstates. A consequence of the fact that the $\mathrm{R}$ ground states (in the usual basis) are eigenstates of the R-charge is that only neutral operators can have non-vanishing expectation values on these states. We compute the holographic 1-point functions of the fuzzball solutions and find that charged chiral primaries have non-zero expectation values, except when the curve characterizing the solution is circular. The non-zero vevs reflect the fact that a generic curve breaks R-symmetry completely. This implies that fuzzball solutions (excepting circular ones) can only correspond to superpositions of $\mathrm{R}$ states and we give a proposal for the superposition corresponding to a given curve. We also address the question of what would be the geometric dual of a given $\mathrm{R}$ ground state.
\end{abstract}

PACS numbers: $11.25 . \mathrm{Tq}$ 04.70.-s $11.25 . \mathrm{w}$

Black holes provide a unique probe of non-perturbative aspects of quantum gravity and as such they have played a prominent role in many important developments over the years. In particular, the fact that they carry entropy and Hawking radiate, along with the associated information loss paradox, imposes restrictions and challenges on any potential quantum theory of gravity. Progress in resolving these issues has been achieved for a class of supersymmetric black holes using string theory. The degeneracy of the microstates of these black holes can be computed from first principles using D-branes. Furthermore, the AdS/CFT correspondence implies that the gravitational evolution is unitary. These developments however have left unanswered the question of what are the black hole microstates in the original gravitational description of the system.

An interesting proposal for the gravitational nature of the microstates was made in [1], see [2] for a review and further references. According to this proposal there should be an exponential number of horizon-free solutions associated to each black hole, one for each microstate (although in general only a subset of these solutions will be realized as good supergravity solutions). The microstates should resemble the original black solution asymptotically and (generically) they should differ from each other up to the horizon scale. The original black hole would provide only the "average" description of the system; the true description is in terms of the nonsingular microstate geometries.

This is a very appealing picture that would resolve long standing puzzles associated with black hole physics such as the information loss paradox. It is thus important to scrutinize the evidence for it and further develop it in a way that quantitative analysis is possible. Such a precise quantitative framework is required for this picture to become a physical model that would ultimately resolve the issues discussed above or be falsified. The main aim

\footnotetext{
${ }^{*}$ The authors are supported by NWO.
}

of this work is to provide such a framework.

A test case for this proposal has been the 2-charge D1D5 system. This is a $1 / 4$ supersymmetric system and the "naive" black hole geometry has a near-horizon geometry of the form $A d S_{3} \times S^{3} \times M$, where $M$ is either $T^{4}$ or $K 3$. Associated with this system an exponential number of horizon-free solutions has been found [1] and proposed to correspond to microstates. These solutions can account for a finite fraction of the black hole entropy. One still needs an exponentially large number of solutions to account for all of the black hole entropy and such solutions were discussed in [4]. In this case the AdS/CFT correspondence provides a dual description of the microstates so one should be able to quantitatively understand the relation between the horizon-free geometries and the actual microstates.

Ultimately the fuzzball picture should apply to any black hole, supersymmetric or not. It would however be an enormous task to find the required number of horizonfree solutions for all cases. Even in the next simplest case, i.e. the $1 / 8$ supersymmetric black holes obtained by adding momentum charge to the D1-D5 system, the current results are very incomplete with only a small number of horizon-free geometries known. From our point of view, one should first understand in detail the precise properties that the required solutions should have and how many of them should be accessible in supergravity before embarking upon such a task. Thus one should first understand completely the D1-D5 system, in particular, how black hole properties emerge upon coarse graining.

The solutions of [1] were found using the fact that the D1-D5 system is related by dualities to a fundamental string carrying momentum. Such F-P solutions were constructed in [5] and carrying out the dualities leads to the following D1-D5 solution

$$
\begin{aligned}
d s^{2}= & f_{1}^{-1 / 2} f_{5}^{-1 / 2}\left(-(d t-A)^{2}+(d y+B)^{2}\right) \\
& +f_{1}^{1 / 2} f_{5}^{1 / 2} d x \cdot d x+f_{1}^{1 / 2} f_{5}^{-1 / 2} d z \cdot d z ; \\
e^{2 \Phi}= & f_{1} f_{5}^{-1} ; \quad C_{t i}=f_{1}^{-1} B_{i} ; \quad C_{t y}=f_{1}^{-1} ; \\
C_{y i}= & f_{1}^{-1} A_{i} ; \quad C_{i j}=c_{i j}-f_{1}^{-1}\left(A_{i} B_{j}-A_{j} B_{i}\right),
\end{aligned}
$$


where the metric is in the string frame and the fields satisfy

$$
\begin{aligned}
& d c=*_{4} d f_{5}, \quad d B=*_{4} d A, \\
& \square_{4} f_{1}=\square_{4} f_{5}=\square_{4} A_{i}=0, \quad \partial^{i} A_{i}=0 .
\end{aligned}
$$

where the Hodge dual $*_{4}$ and $\square_{4}$ are defined on the four (flat) non-compact overall transverse directions $x^{i}$. The compact part of the geometry can be either $T^{4}$ or $K 3$.

The harmonic functions appearing in the solutions of [1] are determined by an arbitrary closed curve $F^{i}(v)$ of length $L$ in $R^{4}$,

$$
\begin{aligned}
& f_{5}=1+\frac{Q_{5}}{L} \int_{0}^{L} \frac{d v}{|x-F|^{2}} ; f_{1}=1+\frac{Q_{5}}{L} \int_{0}^{L} \frac{d v|\dot{F}|^{2}}{|x-F|^{2}} \\
& A_{i}=\frac{Q_{5}}{L} \int_{0}^{L} \frac{\dot{F}_{i} d v}{|x-F|^{2}},
\end{aligned}
$$

The physical interpretation of these solutions is that the D1 and D5 brane sources are distributed on a curve in the transverse $R^{4}$. The D5-branes are uniformly distributed along this curve, but the D1-brane density at any point on the curve depends on the tangent to the curve, $Q_{1}=\left(Q_{5} / L\right) \int_{0}^{L}|\dot{F}|^{2} d v$, where the $Q_{i}$ are related to the integral charges by $Q_{1}=\left(\alpha^{\prime}\right)^{3} n_{1} / V, Q_{5}=\alpha^{\prime} n_{5}$, with $(2 \pi)^{4} V$ the volume of the compact manifold (we set the string coupling constant to one, $g_{s}=1$ ). Furthermore, the length of the curve is given by $L=2 \pi Q_{5} / R$, where $R$ is the radius of the $y$ circle. Finally, we require that the curve satisfies $\int d v F^{i}\left(1+\left(Q_{5} / Q_{1}\right)|\dot{F}|^{2}\right)=0$. This corresponds to choosing the origin of the coordinate system to be at the center of mass of the system.

It was argued in [1] that these solutions are related to the $\mathrm{R}$ ground states and via spectral flow also to chiral primaries common to both the $T^{4}$ and $K 3$ boundary CFTs [3]. Roughly speaking, the proposal was that different curves correspond to different $\mathrm{R}$ ground states/chiral primaries. We will show in this letter that only the circular curves can correspond to $\mathrm{R}$ ground states/chiral primaries (in the usual basis) and we will propose a precise map between all geometries and superposition of $\mathrm{R}$ ground states. We further address the question of what would be the geometric dual of a given $\mathrm{R}$ ground state.

The low energy dynamics of the D1-D5 system is governed by a $2 \mathrm{~d}$ CFT with $\mathcal{N}=(4,4)$ supersymmetry. The internal R-symmetry is $S U(2)_{L} \times S U(2)_{R}$, with $S U(2)_{L}$ carried by left movers and $S U(2)_{R}$ by right movers. The detailed structure of the CFT will not be important in our discussion; we will only need some general facts about the chiral primaries and the associated $\mathrm{R}$ ground states that we now review. The chiral primaries in the NS sector are states with $h^{N S}=j_{3}^{N S}, \bar{h}^{N S}=\bar{j}_{3}^{N S}$, where $\left(h^{N S}, \bar{h}^{N S}\right)$ are the conformal dimensions and $j_{3}^{N S}$ and $\bar{j}_{3}^{N S}$ are the $U(1) \times U(1)$ charges (of the Cartan of $\left.S U(2)_{L} \times S U(2)_{R}\right)$ for the left and right moving sectors, respectively. For each chiral primary there is a corresponding $\mathrm{R}$ ground state obtained via spectral flow, with
$h^{R}=h^{N S}-j_{3}^{N S}+c / 24, \quad j_{3}^{R}=j_{3}^{N S}-c / 12$. The Rground states thus obtained have the same dimension and definite R-charges.

Consider now the expectation value of an operator $O^{k_{1}, k_{2}}$ of charge $j_{3}^{R}=k_{1}, \bar{j}_{3}^{R}=k_{2}$ in a $\mathrm{R}$ ground state. Charge conservation implies that this expectation value is zero, unless the operator is neutral under $U(1) \times U(1)$,

$$
\left\langle-j_{3}^{R},-\bar{j}_{3}^{R}\left|O^{k_{1}, k_{2}}\right| j_{3}^{R}, \bar{j}_{3}^{R}\right\rangle=0, \quad\left\{k_{1} \neq 0 \text { or } k_{2} \neq 0\right\}
$$

where we label the $\mathrm{R}$ ground states by their $\mathrm{R}$ charge and use the fact that the bra state has the opposite $\mathrm{R}$ charge to the ket state. It follows that for a geometry to be dual to a specific $\mathrm{R}$ ground state the holographic 1-point functions of all charged operators must be equal to zero.

In the decoupling limit, one may drop the one in the harmonic functions (3) and the fuzzball solutions become asymptotically $A d S_{3} \times S^{3}$. One can then use the AdS/CFT correspondence to compute the expectation value of $1 / 2$ BPS operators of the dual CFT. A detailed analysis of this computation appears in a companion paper [6]; here we will only present the results for a few relevant operators.

Recall that in the AdS/CFT correspondence the boundary fields parametrizing the Dirichlet boundary conditions of supergravity fields are identified with sources of boundary gauge invariant operators [7]. Thus to obtain the vev of a given operator we need to compute the variation of the on-shell action w.r.t. the corresponding source. This computation requires appropriate renormalization and results in a concrete formula for the vev in terms of certain coefficients in the asymptotic expansion of the bulk fields [8] (see [9] for a review and further references). A precise holographic map between the vevs of $1 / 2$ BPS operators and asymptotically $A d S \times S$ solutions was constructed in our recent work [10]. This construction starts by decomposing the deviation of the fuzzball metrics from $A d S_{3} \times S^{3}$ in $S^{3}$ harmonics and performing a radial expansion of the resulting coefficients.

The six dimensional theory obtained by reducing the ten dimensional solutions over the internal manifold involves the metric $g_{M N}$, the dilaton $\Phi$ and the 3 -form (which we decompose into self-dual and anti-delf dual parts) $G^{( \pm)}=1 / 2(1 \pm *) d C$. Let us denote the deviations of the six dimensional solution from the $A d S_{3} \times S^{3}$ solution by $\left(h_{M N}, g_{M N P}^{( \pm)}, \phi\right)$, where $M=\{\mu, a\}$ and $\mu$ and $a$ are $A d S_{3}$ and $S^{3}$ indices, respectively. The harmonic expansion of these deviations contains many terms; here we only give the terms relevant for us

$$
\begin{aligned}
h_{\mu a} & =\sum h_{\mu}^{I_{v} \pm}(x) Y_{a}^{I_{v} \pm}(y)+\cdots \\
h_{(a b)} & =\sum \rho^{I}(x) D_{(a} D_{b)} Y^{I}(y)+\cdots \\
h_{a}^{a} & =\sum \pi^{I}(x) Y^{I}(y) \\
g_{\mu a b}^{( \pm)} & =\sum\left(D_{\mu} U^{( \pm) I} \epsilon_{a b c} D^{c} Y^{I}+2 Z_{\mu}^{( \pm) I_{v} \pm} D_{[b} Y_{a]}^{I_{v} \pm}\right) \\
\phi & =\sum \phi^{I}(x) Y^{I}(y),
\end{aligned}
$$


where $x$ and $y$ are $A d S_{3}$ and $S^{3}$ coordinates, parenthenses denote symmetrization with the trace removed, and $Y^{I}$ and $Y_{a}^{I_{v} \pm}$ are scalar and vector harmonics of $S^{3}$, with the degree $k$ vector harmonics satisfying (apart from the usual eigenvalue equation) $\epsilon_{a b c} D^{b} Y^{c I_{v} \pm}= \pm(k+1) Y_{a}^{I_{v} \pm}$.

The spectrum of the KK excitations was computed in [11] and was matched to the spectrum of $1 / 2$ BPS operators of the dual CFT in [12]. In particular, the KK spectrum consists of a tower of spin two supermultiplets, along with two towers of spin 1 supermultiplets, one coming from combinations of the scalar and the anti-self dual tensor field and the second coming from the metric and self-dual tensor field. The lowest lying chiral primaries of these two towers are $S_{j}^{1}, \Sigma_{I}^{2}$ and they correspond respectively to 4 scalar operators $O_{S_{j}^{1}}(j=1, \ldots, 4)$ of dimension 1 transforming in the $(1 / 2,1 / 2)$ representation of the R-symmetry $S O(4)$, and 9 scalar operators $O_{\Sigma_{I}^{2}}$ of dimension 2 transforming in the $(1,1)$ representation. We will also be interested in the R-symmetry current $j_{u}^{ \pm \alpha}$, where $\alpha$ is an $S U(2)$ adjoint index and $u$ a boundary spacetime index. This corresponds to the gauge field $A_{u}^{ \pm \alpha}$ coming from the spin two tower.

The vacuum expectation values of these operators can be obtained from the asymptotics of the coefficients in (5). The derivation of these (and other) holographic 1point functions is discussed in detail in [6]. To compute the explicit value of the vevs we need to expand asymptotically the solution in (1). The (near-horizon) harmonic functions appearing in the solution can be expanded as

$$
\begin{aligned}
& f_{5}=\frac{Q_{5}}{r^{2}} \sum_{k, I} \frac{f_{k I}^{5} Y_{k}^{I}\left(\theta_{3}\right)}{r^{k}} ; \quad f_{1}=\frac{Q_{1}}{r^{2}} \sum_{k, I} \frac{f_{k I}^{1} Y_{k}^{I}\left(\theta_{3}\right)}{r^{k}} ; \\
& A_{i}=\frac{Q_{5}}{r^{2}} \sum_{k \geq 1, I} \frac{\left(A_{k I}\right)_{i} Y_{k}^{I}\left(\theta_{3}\right)}{r^{k}}
\end{aligned}
$$

where $\left(r, \theta_{3}\right)$ are polar coordinates in the overall transverse $R^{4}$ and we explicitly indicate the degree of the harmonics $k$. The coefficient $f_{k I}^{5}, f_{k I}^{1},\left(A_{k I}\right)_{i}$ for the harmonic functions in (3) will be given below; for now we proceed in general. Inserting these expansions into the solution and extracting the relevant coefficients leads after some manipulations to

$$
\begin{aligned}
& \left\langle j^{ \pm \alpha}\right\rangle= \pm \frac{N}{2 \pi}\left(A_{1 j}\right)_{i} e_{\alpha i j}^{ \pm} d y^{\mp}, \quad\left\langle O_{S_{i}^{1}}\right\rangle=-\frac{N \sqrt{2}}{\pi} f_{1 i}^{5} ; \\
& \left\langle O_{\Sigma_{I}^{2}}\right\rangle=\frac{N \sqrt{2}}{4 \pi}\left(-\left(f_{2 I}^{1}+f_{2 I}^{5}\right)+\left(A_{1 j}\right)_{i}\left(A_{1 l}\right)_{k} e_{i j k l}^{I}\right), \quad(7)
\end{aligned}
$$

where $y^{ \pm}=y \pm t, I \equiv i$ and $I_{v} \equiv \alpha$ for degree 1 harmonics, $e_{i j k l}^{I}=8 f_{\alpha \beta}^{I} e_{\alpha i j}^{+} e_{\beta k l}^{-}, \quad f_{\alpha \beta}^{I}=1 /\left(2 \pi^{2}\right) \int Y^{I}\left(Y^{\alpha-}\right)^{a}\left(Y^{\beta+}\right)_{a}$ and $e_{\alpha i j}^{ \pm}=\sqrt{Q_{5} / Q_{1}} /\left(2 \pi^{2}\right) \int\left(Y^{\alpha \mp}\right)^{b} Y^{j} \partial_{b} Y^{i}$. Notice that the vevs of the operators of dimension 1 depend linearly on the degree 1 coefficients in (6) and the vevs of the dimension 2 operators depend linearly on the degree 2 coefficients and quadratically on the degree 1 coefficients. This is the structure of the vevs of all chiral primary operators, i.e. the vev depends linearly on the degree $k$ coefficient, where $k$ is the dimension of the operator, and non-linearly on lower degree coefficients but such that the sum of their degrees is $k$. One can easily obtain the linear terms but computation of the coefficients of the non-linear terms is very tedious in practice, although it is in principle possible, and has only been done up to dimension 2 operators [6]. This concludes our general discussion of the vevs associated with the solution (1).

We now consider the solutions specified by a closed curve $F^{i}$. In this case the coefficients (relevant for us) in (6) are given by

$$
\begin{aligned}
f_{k I}^{5} & =\frac{1}{(k+1) L} \int_{0}^{L} d v C_{i_{1} \cdots i_{k}}^{I} F^{i_{1}} \cdots F^{i_{k}} \\
f_{k I}^{1} & =\frac{Q_{5}}{Q_{1}(k+1) L} \int_{0}^{L} d v|\dot{F}|^{2} C_{i_{1} \cdots i_{k}}^{I} F^{i_{1}} \cdots F^{i_{k}} ; \\
\left(A_{1 j}\right)_{i} & =\frac{1}{2 L} \int_{0}^{L} d v \dot{F}_{i} F_{j} .
\end{aligned}
$$

where $C_{i_{1} \cdots i_{k}}^{I}$ are the orthogonal symmetric traceless rank $k$ tensors on $R^{4}$.

The operators $O_{S_{i}^{1}}$ and all but one of the operators $O_{\Sigma_{I}^{2}}$ are charged under the Cartan of $S O(4)$. It follows that for the solutions to correspond to a $\mathrm{R}$ ground state the vevs of these operators must vanish. Before discussing the general case, let us consider the case of an ellipsoidal planar curve in the 1-2 plane,

$$
F^{1}=\frac{\mu \alpha}{n} \cos \frac{2 \pi n v}{L}, \quad F^{2}=\frac{\mu \beta}{n} \sin \frac{2 \pi n v}{L},
$$

where $\mu=\sqrt{Q_{1} Q_{5}} / R$ and $(\alpha, \beta)$ are parameters satisfying $\alpha^{2}+\beta^{2}=2$. Evaluating the vevs of the charged scalar operators we find the following non-zero vevs,

$$
\left\langle O_{\Sigma^{2}}^{( \pm 2,0)}\right\rangle=-\frac{\sqrt{3} N}{8 \sqrt{2} \pi} \frac{\mu^{2}}{n^{2}}\left(\alpha^{2}-\beta^{2}\right)
$$

where we have explicitly indicated the $U(1) \times U(1)$ charges $\left(j_{3}^{R}+\bar{j}_{3}^{R}, j_{3}^{R}-\bar{j}_{3}^{R}\right)$. The vev of the R-symmetry current is $\left\langle j^{ \pm 3}\right\rangle=(N / 4 \pi)(\mu / n) \alpha \beta d y^{\mp}$.

We thus find that only for circles (with $\alpha^{2}=\beta^{2}=1$ )) do the vevs of charged operators vanish and therefore only these geometries can correspond to $\mathrm{R}$ ground states. One might have anticipated this result: a planar ellipse breaks the $S O(4)$ R-symmetry to $S O(2)$ (times certain discrete transformations) and this is reflected in the vev of $O_{\Sigma^{2}}^{( \pm 2,0)}$. The vanishing of the vevs of $O_{S_{i}^{1}}$ and of the other 6 charged operators $O_{\Sigma^{2}}$ is due to the preserved $S O(2)$ and discrete symmetries. It is then clear that the only geometries that have vanishing vevs for all charged operators are the ones that have an $S O(2) \times S O(2)$ symmetry. The only such solutions are the circular ones. (There may be non-circular curves with $f_{1 i}^{5}=f_{2 I}^{1}=$ $f_{1 I}^{5}=0$ so that all vevs in (7) are zero but in this case one would find non-zero vevs of higher dimension charged operators characterizing the symmetry breaking). 
Thus we arrive at the conclusion that of all fuzzball solutions only the circular ones can correspond to $\mathrm{R}$ ground states. This seems to be at odds with the fact that all solutions (1)-(3) are related by dualities [1] to solutions that are believed to correspond to strings carrying momentum and thus should be related to bound states of D1 and D5 branes. This argument, however, only implies that each geometry should be related to some linear superposition of $\mathrm{R}$ ground states. Indeed, the vevs of charged operators in a state that is not an eigenstate of the R-charge are allowed to be non-zero.

We now propose a map between fuzzball geometries and superpositions of $\mathrm{R}$ ground states, which passes all kinematical and all accessible dynamical tests. The motivation for this map and various tests are discussed in detail in [6]. Given the curve $F^{i}(v)$ which defines the fuzzball geometry, we construct a corresponding coherent state in the dual FP system and then find which Fock states in this coherent state satisfy the constraint $N_{L}=N$, where $N_{L}$ is the left moving excitation number. Mapping these states to the D1-D5 system, corresponding fundamental string oscillators to $\mathrm{R}$ operators, yields the superposition of ground states which is proposed to be dual to the D1-D5 geometry.

In particular, for the case of the ellipse in (9), the conjectured superposition is |ellipse) $=$ $\sum_{k=0}^{N / n} 2^{-N / n} \sqrt{C(N / n, k)}(\alpha+\beta)^{\frac{N}{n}-k}(\alpha-\beta)^{k} \mathcal{O}_{k}^{R}$, where $C(N / n, k)$ is the bimomial coefficient and $\mathcal{O}_{k}^{R}$ are particular R ground states with $\mathrm{R}$ charge $j_{3}^{R}=\bar{j}_{3}^{R}=(N / 2 n-k)$. This proposal passes all kinematical checks and yields the correct answer for the vev of the R-symmetry current quoted above. The field theory computation of the vev in (10) as well as the vevs of other neutral and charged operators would require a knowledge of certain multiparticle three point functions at strong coupling, which are not known, but approximating these leads to vevs which agree remarkably well with those extracted from supergravity. In particular, one recovers (10) up to an overall numerical factor.

This then leaves the question: "what are the geome- tries that are dual to R ground states"? As has already been discussed, a geometry which is dual to a $\mathrm{R}$ ground state must preserve the $S O(2) \times S O(2)$ symmetry. This immediately implies that the asymptotics must be of the following form:

$$
\begin{aligned}
& f_{5}=\frac{Q_{5}}{r^{2}} \sum_{k=2 l} \frac{f_{k 0}^{5}}{r^{k}} Y_{k}^{0} ; \quad f_{1}=\frac{Q_{1}}{r^{2}} \sum_{k=2 l} \frac{f_{k 0}^{1}}{r^{k}} Y_{k}^{0} \\
& A_{a}=\sum_{k} \frac{Q_{5}}{r^{k+1}}\left(A_{k 0+} Y_{k a}^{0+}+A_{k 0-} Y_{k a}^{0-}\right)
\end{aligned}
$$

where the scalar spherical harmonics $Y_{2 l}^{0}$ are singlets under $S O(2) \times S O(2)$, and $Y_{k a}^{0 \pm}$ are vector spherical harmonics of degree $k$ ( $k$ odd) whose Lie derivatives along the $S O(2)^{2}$ directions are zero; note that these forms have only components along the $(\phi, \psi)$ directions.

Several examples of exact solutions which have such asymptotics are discussed in [6]. One class of such solutions can be obtained by superimposing harmonic functions, sourced on the $S O(2) \times S O(2)$ orbits of a given curve. Another class of solutions is obtained using disconnected circular curves, although one would expect that such solutions do not correspond to Higgs branch vacua. All of these solutions pass the kinematical constraints to be identified as the dual of $\mathrm{R}$ ground states (or chiral primaries after spectral flow). To distinguish between them, one will thus need to use dynamical information, namely the actual non-zero values of the vevs of neutral operators. This is a very subtle issue which will in general require going beyond the leading supergravity approximation, as we discuss in [6].

In conclusion, our results support the overall fuzzball picture: the solutions can be in correspondence with the black hole microstates in a way that is compatible with the AdS/CFT correspondence. The detailed correspondence however is more complicated than anticipated. Furthermore, even in the simplest 2-charge system one would need to go beyond the leading supergravity approximation to test any proposed correspondence.
[1] O. Lunin and S. D. Mathur, Nucl. Phys. B 623, 342 (2002), hep-th/0109154.

[2] S. D. Mathur, hep-th/0502050.

[3] O. Lunin, S. D. Mathur and A. Saxena, Nucl. Phys. B 655 (2003) 185, hep-th/0211292.

[4] O. Lunin, J. Maldacena and L. Maoz, hep-th/0212210; M. Taylor, JHEP 0603 (2006) 009, hep-th/0507223.

[5] C. G. Callan, J. M. Maldacena and A. W. Peet, Nucl. Phys. B 475, 645 (1996) [arXiv:hep-th/9510134]; A. Dabholkar, J. P. Gauntlett, J. A. Harvey and D. Waldram, Nucl. Phys. B 474, 85 (1996), hep-th/9511053.

[6] I. Kanitscheider, K. Skenderis and M. Taylor, hepth/0611171.

[7] S. S. Gubser, I. R. Klebanov and A. M. Polyakov, Phys.
Lett. B 428, 105 (1998), hep-th/9802109; E. Witten, Adv. Theor. Math. Phys. 2, 253 (1998), hep-th/9802150.

[8] S. de Haro, S. N. Solodukhin and K. Skenderis, Commun. Math. Phys. 217, 595 (2001), hep-th/0002230,

[9] K. Skenderis, Class. Quant. Grav. 19, 5849 (2002), hepth/0209067.

[10] K. Skenderis and M. Taylor, JHEP 0605, 057 (2006), hep-th/0603016.

[11] S. Deger, A. Kaya, E. Sezgin and P. Sundell, Nucl. Phys. B 536, 110 (1998), hep-th/9804166.

[12] J. de Boer, Nucl. Phys. B 548, 139 (1999), hepth/9806104. 